\title{
Non-adiabatic spin-torques in narrow magnetic domain walls
}

\author{
C. Burrowes ${ }^{1,2}$, A. P. Mihai ${ }^{3,4}$, D. Ravelosona ${ }^{1,2 \star}$, J.-V. Kim ${ }^{1,2}$, C. Chappert ${ }^{1,2}$, L. Vila ${ }^{3,4}$, A. Marty ${ }^{3,4}$, \\ Y. Samson ${ }^{3}$, F. Garcia-Sanchez ${ }^{5}$, L. D. Buda-Prejbeanu ${ }^{5}$, I. Tudosa ${ }^{6}$, E. E. Fullerton ${ }^{6}$ and J.-P. Attané ${ }^{3,4}$
}

Torques appear between charge carrier spins and local moments in regions of ferromagnetic media where spatial magnetization gradients occur, such as a domain wall, owing to an exchange interaction. This phenomenon has been predicted by different theories ${ }^{1-7}$ and confirmed in a number of experiments on metallic and semiconductor ferromagnets ${ }^{8-19}$. Understanding the magnitude and orientation of such spin-torques is an important problem for spin-dependent transport and currentdriven magnetization dynamics, as domain-wall motion underlies a number of emerging spintronic technologies ${ }^{20,21}$. One outstanding issue concerns the non-adiabatic spin-torque component $\beta$, which has an important role in wall dynamics, but no clear consensus has yet emerged over its origin or magnitude. Here, we report an experimental measurement of $\beta$ in perpendicularly magnetized films with narrow domain walls (1-10 nm). By studying thermally activated wall depinning, we deduce $\beta$ from the variation of the Arrhenius transition rate with applied currents. Surprisingly, we find $\beta$ to be small and relatively insensitive to the wall width, which stands in contrast to predictions from transport theories ${ }^{2,5-7}$. In addition, we find $\beta$ to be close to the Gilbert damping constant $\alpha$, which, in light of similar results on planar anisotropy systems ${ }^{15}$, suggests a universal origin for the non-adiabatic torque.

The adiabatic torque, which accounts for transport processes in which the conduction spin follows the local spatial magnetization variation by remaining in either the majority or minority state, is well understood and has been reproduced by a number of different transport theories. In contrast, the non-adiabatic contribution, characterized by a dimensionless parameter $\beta$ (ref. 22), remains the subject of much debate. Various mechanisms have been put forward to explain its origin, such as momentum transfer ${ }^{2,7}$, spinmistracking ${ }^{4,6}$ or spin-flip scattering ${ }^{3}$. It is predicted that large nonadiabatic effects should appear in narrow domain walls because of large magnetization gradients ${ }^{2,5,6}$, whereby the wall width becomes comparable to important transport scales such as the spin-diffusion length ${ }^{2}$ or the Larmor precession length ${ }^{6}$, which are of the order of a few nanometres in ferromagnetic transition metals. The presence of a non-adiabatic term is of fundamental importance, because its existence implies that current-driven wall motion is possible for any finite current in a perfect system, even in the absence of an applied magnetic field. Difficulty in characterizing $\beta$ experimentally therefore stems in part from being able to distinguish between extrinsic sources of wall pinning, due to structural defects, for example, from the intrinsic finite threshold current predicted ${ }^{2}$ for $\beta=0$.

We have studied current-driven domain wall dynamics in two different pseudo spin-valve systems based either on CoNi
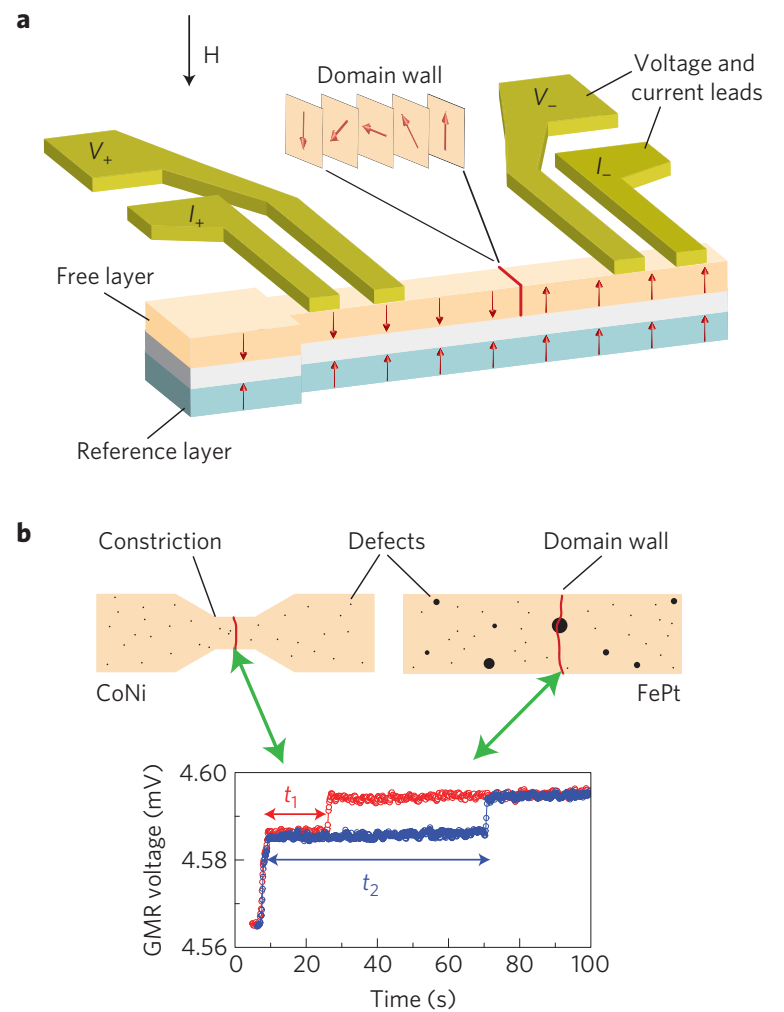

Figure 1 | Schematic overview of experiment. a, Domain-wall propagation is detected in the free layer of a spin valve by measuring the in-plane giant magnetoresistance between the $V_{+}$and $V_{-}$leads as a function of time. The a.c. (lock-in detection of the giant magnetoresistance voltage) and d.c. current are injected between leads $I_{+}$and $I_{-}$. Typical wire dimensions are $10 \mu \mathrm{m}$ long and $200 \mathrm{~nm}$ wide. $\mathbf{b}$, The statistics of thermally activated domain-wall depinning are determined using a large intrinsic defect in FePt and a constriction in CoNi. An example of a time-resolved giant magnetoresistance (GMR) measurement is shown. The lowest (highest) resistance is reached when the domain wall passes the first (second) electrical contact. Between these two values, the variation of the giant magnetoresistance as a function of time corresponds to domain-wall motion between the two contacts. The motion occurs in a series of two abrupt jumps separated by long plateaux where the wall is pinned. The two different pinning times obtained at the same magnetic field and current illustrate the stochastic nature of domain-wall depinning under currents.

\footnotetext{
${ }^{1}$ Institut d'Electronique Fondamentale, CNRS, UMR 8622, 91405 Orsay, France, ${ }^{2}$ Université Paris-Sud, 91405 Orsay, France, ${ }^{3}$ CEA, Inac, SP2M, 38054 Grenoble, France, ${ }^{4}$ Université Joseph Fourier, 38041 Grenoble, France, ${ }^{5}$ SPINTEC, URA2512 CEA/CNRS/UJF/INPG, 38054 Grenoble, France, ${ }^{6}$ Center for Magnetic Recording Research, University of California at San Diego, La Jolla, California 92093-0401, USA. *e-mail: dafine.ravelosona@u-psud.fr.
} 

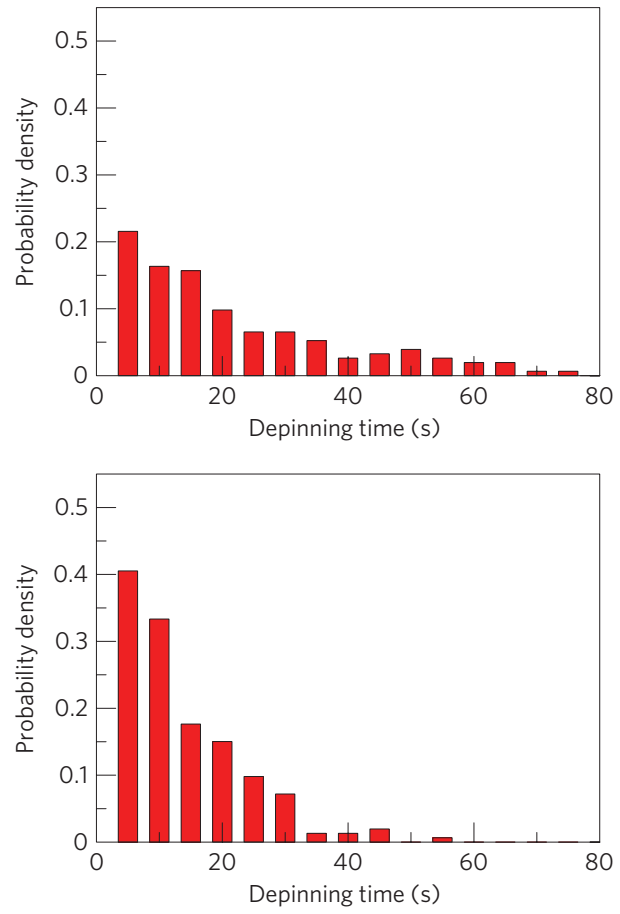

b

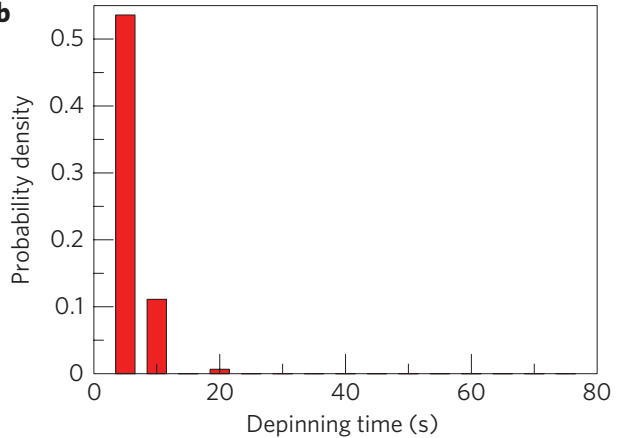

d

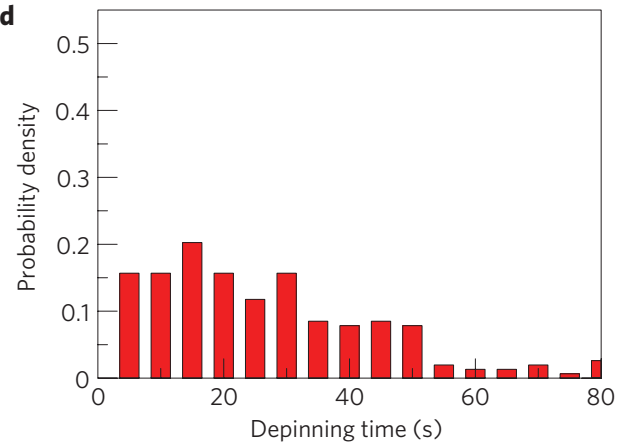

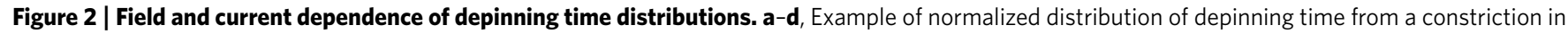
a CoNi system for $H=550 \mathrm{Oe}, J=0(\mathbf{a}) ; H=590 \mathrm{Oe}, J=0(\mathbf{b}) ; H=550 \mathrm{Oe}, J^{\prime}=10^{10} \mathrm{~A} \mathrm{~cm}^{-2}(\mathbf{c}) ; H=550 \mathrm{Oe}, \mathrm{J}=-10^{10} \mathrm{~A} \mathrm{~m}^{-2}$ (d). In our convention, positive currents favour wall motion in the same direction as a positive applied field, and vice versa.

multilayers $^{18}$ or FePt alloy thin films ${ }^{23,24}$, which possess large magnetocrystalline anisotropies that lead to narrow domain walls $(\lambda=10 \mathrm{~nm}$ in CoNi and $1 \mathrm{~nm}$ in $\mathrm{FePt})$. In these materials, the uniaxial anisotropy is perpendicular to the film plane, which results in simple Bloch wall structures. Figure 1a shows a schematic diagram of our film structures and geometry used. The free magnetic layer in which the domain wall propagates is composed of a $[\mathrm{Co}(0.15) / \mathrm{Ni}(0.6)]_{4} / \mathrm{Co}(0.3) / \mathrm{Pd}(0.7)$ multilayer for the $\mathrm{CoNi}$ system and a highly chemically ordered $\mathrm{L}_{10} \mathrm{FePt}(4)$ film for the FePt system, where the figures in parentheses denote the film thicknesses in nanometres and the subscript indicates the number of repetitions. The stacks are patterned using electronbeam lithography and ion etching into 200-nm-wide wires to conduce wall motion in only one dimension. A large magnetic pad is defined at one wire end, which allows domain walls to be nucleated and injected into the wire. One set of electrical contacts at the wire ends allows a.c. and d.c. electrical currents to be applied to detect and drive respectively the domain wall, while a separate set of contacts allows the time-varying in-plane giant magnetoresistance signal to be measured simultaneously to track the domain-wall position. As illustrated in Fig. 1b, for the CoNi system, we have used lithographically defined constrictions along the wire to pin the domain wall at specified positions, whereas for the FePt system the domain wall is reproducibly pinned on a single natural defect with a pinning strength that dominates those of other pinning sites within the nanowire ${ }^{23,24}$. From hysteresis loop measurements, carried out at a sweep rate of $10 \mathrm{Oes}^{-1}$ at room temperature, we find a depinning field (averaged over 20 measurements) of $0.58 \mathrm{kOe}$ for the CoNi system and $4.5 \mathrm{kOe}$ for the FePt system. We determine $\beta$ experimentally using the statistics of thermally activated domain-wall depinning from these pinning centres.

Figure 2 shows several distributions of pinning times measured as a function of applied field and current. For each measurement, the domain wall is nucleated in the reservoir and propagates in the wire under field and current until it is pinned at the constriction $(\mathrm{CoNi})$ or on the natural defect $(\mathrm{FePt})$. Meanwhile, the giant magnetoresistance effect is monitored as a function of time (Fig. 1b). After a certain duration, termed the pinning time, thermal activation causes the wall to depin from the pinning site and propagate farther along the wire until it is annihilated at the end of the wire (corresponding to full magnetization reversal of the free layer). This procedure is repeated several hundred times for each applied field and current. When the applied field is increased, the distribution of pinning times becomes narrower (Fig. 2a,b), which reflects a more deterministic dynamics as the energy barrier at the pinning centre is reduced. As expected, the opposite behaviour is observed when the applied field is decreased, whereby the spread in pinning times becomes larger. This asymmetry is also observed for weak applied d.c. currents (well below the critical current needed to drive wall motion in zero field), as shown in Fig. 2, whereby positive currents (Fig. 2c) lead to narrower distributions and negative currents (Fig. 2d) to wider ones. This observation confirms the presence of spin-transfer, as current-induced heating would lead to a decrease in the energy barrier for both current polarities.

The range of depinning times measured is limited by a number of experimental constraints (see Supplementary Information). The lower bound is determined by the rise time of the electromagnet, which restricts the strength of the magnetic field applied; it takes $0.5-1 \mathrm{~s}$ to obtain fields in excess of $1 \mathrm{kOe}$. Therefore, it is possible to obtain a good estimate of the depinning time only if the propagation time of the domain wall from the nucleation reservoir exceeds this rise time. The upper bound is determined by problems related to electrical discharge. Electrical discharge shortens the lifetime of the samples and also places an upper limit on the range of currents we can apply. As a result of the statistical nature of the experiment, whereby several hundreds of measurements are made for each applied field and current, each depinning time measurement is limited to $120 \mathrm{~s}$ to avoid premature device breakdown.

Thermal activation over a single energy barrier is described by an Arrhenius law, whereby the probability of escape over the barrier $E_{\mathrm{b}}$ is exponential and characterized by a time constant $\tau=\tau_{0} \exp \left(E_{\mathrm{b}} / k_{\mathrm{B}} T\right)$, with an attempt frequency $\tau_{0}^{-1}$ (refs 24, 25). 
a

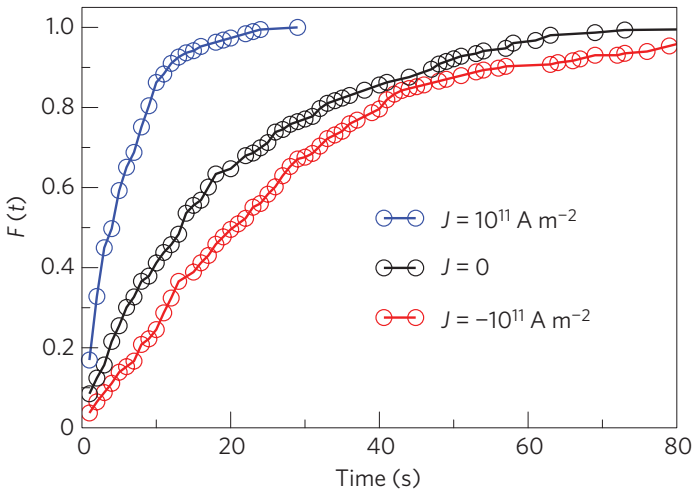

b

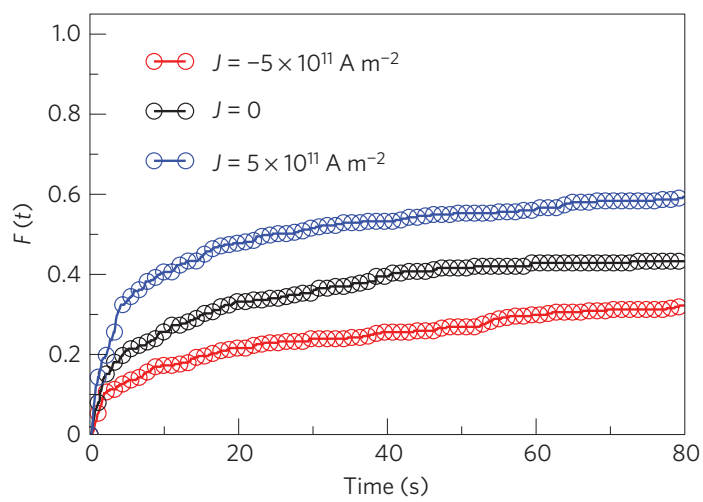

Figure 3 | Cumulative distribution functions. a,b, Cumulative probability function $F(t)$ of depinning at constant magnetic field for zero, positive and negative current for $\mathrm{CoNi}(\mathbf{a})$ and $\mathrm{FePt}(\mathbf{b})$. The $F(t)$ functions were obtained by integrating the distribution of depinning time (Fig. 2).

We use the cumulative distribution function, $F(t)=1-\exp (-t / \tau)$, which is obtained by integrating over the measured distribution of pinning times. Example $F(t)$ results are shown in Fig. 3. For the CoNi system, we have verified that $F(t)$ fits well with an exponential function (Fig. 3a), which is consistent with a depinning process dominated by a single energy barrier. From these fits, we obtain a measure of the Arrhenius time $\tau$ as a function of field and current. For FePt, we observe that depinning on the main defect is associated with two different mechanisms, each involving a different energy barrier height, similarly to what has been observed previously in FePt single layers ${ }^{24}$ and for artificial defects ${ }^{17}$. For one mechanism the pinning strength is large and leads to a time constant too long to be measured, whereas the other configuration leads to a characteristic time of the order of seconds. Therefore, the function $F(t)$ does not asymptotically approach 1 for the FePt system as $t$ increases (Fig. 3b). Nevertheless, we can isolate the time constant corresponding to the most probable depinning transition $^{24}$, which we use in the following to characterize the spin-torque contribution.

Figure 4 shows the variation of the Arrhenius time $\tau$ as a function of low current densities, under different applied fields. For both systems, we observe a clear linear current dependence for $\ln \tau$, implying a linear reduction in the barrier height. These results can be explained by a theory we have developed to account for spin-torques in thermally activated domain-wall depinning ${ }^{26}$. The key result is that the current-dependent energy barrier has the form $E_{\mathrm{b}}=E_{\mathrm{b}, 0}-\sigma I$, where the efficiency parameter $\sigma$ (with dimensions of J/A in SI units) is proportional to $\beta$ (see the Methods section). The linear variation of the $\ln \tau$ as a function of current has also been confirmed in micromagnetics simulations in which we considered wall depinning from a single defect. By taking into account the measured Joule heating in our fits of the experimental data, we
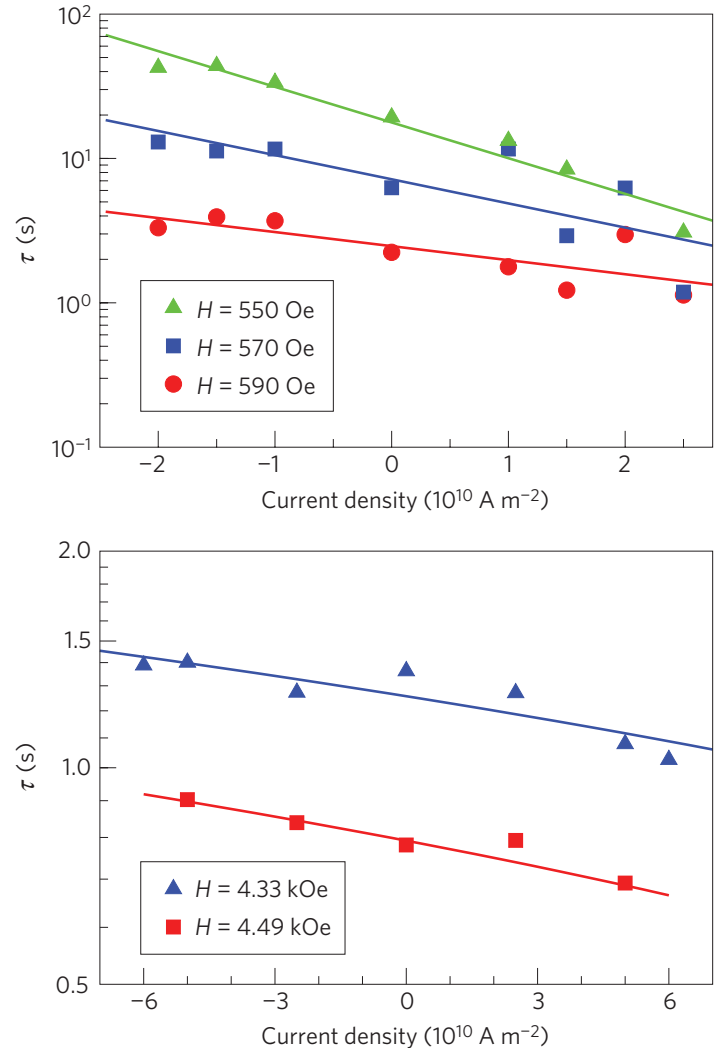

Figure 4 | Current dependence of Arrhenius rate. a,b, Characteristic time $\tau$ as a function of the applied d.c. current density for different magnetic field values for $\mathrm{CoNi}$ (a) and FePt (b). The symbols represent experimental data and the solid lines represent a linear fit on a log scale.

obtain $\beta_{\mathrm{CoNi}}=0.022 \pm 0.002$ and $\beta_{\mathrm{FePt}}=0.06 \pm 0.03$. We note that these values are similar to known values of the Gilbert damping constant, $\alpha_{\mathrm{CoNi}}=0.032 \pm 0.006$ and $\alpha_{\mathrm{FePt}} \simeq 0.1$.

Both the adiabatic and non-adiabatic terms lead to domain-wall displacement, but only the non-adiabatic term has the appropriate symmetry to drive steady-state wall motion as in the case of field-driven dynamics ${ }^{22}$. For this reason, a number of researchers have sought to characterize non-adiabatic torques by associating these as an additional effective field. Our experimental and theoretical results are consistent with this approach in the limit where the barrier height also varies linearly with applied field, but also highlight the limitations of this analogy for perpendicular anisotropy media. First, wall motion in such materials is dominated by strong intrinsic pinning, which may lead to propagation fields that greatly exceed the Walker breakdown field above which wall motion is oscillatory ${ }^{27}$. As such, it is unclear whether a field-current equivalence should be made on the basis of wall motion in the streaming or Walker regime. Second, propagation fields themselves are temperature dependent and are subject to magnetic viscosity, which mean they depend strongly on the sweep rate of applied fields. The latter is exacerbated by Joule heating, which can result in temperature changes of a few hundred kelvin. Our method and analysis accounts explicitly for activation-driven processes, which circumvent the problems highlighted here and lead to a more accurate determination of $\beta$. Whereas the values of $\beta$ we find are much smaller than previous observations on other perpendicular anisotropy systems ${ }^{19}$, the extrapolated critical currents for zero-field currentdriven wall motion are similar. We believe this discrepancy is due to how the current-field equivalence is interpreted for the non-adiabatic torques. 
Our result, that $\beta \approx \alpha$ for walls of 1 and $10 \mathrm{~nm}$ in width, shows that non-adiabatic effects are weak even for narrow walls down to near-atomic dimensions. This is remarkable given that strong non-adiabatic effects are expected on length scales over which spin transport is conserved while large magnetization gradients occur. The fact that $\beta \approx \alpha$ is also observed for wide walls ${ }^{15}$ points towards a universal origin for $\beta$ that may not necessarily be associated with transport processes per se. For example, a recent calculation shows that magnon emission by the domain wall can lead to the appearance of a non-adiabatic term, with $\beta=\alpha$, but only with adiabatic torques ${ }^{28}$. Other theories, which invoke symmetry arguments ${ }^{29}$ or describe spin-flip scattering ${ }^{7}$ leading to both magnetic damping and spin-torques, also predict $\beta=\alpha$. This suggests that non-adiabaticity may be strongly tied to magnetic dissipation processes in general, thereby offering a guide for both future theoretical studies and device engineers who seek to optimize current-driven wall motion for potential spintronic applications $\mathrm{s}^{21,30}$.

\section{Methods}

The adiabatic and non-adiabatic spin-torque terms refer to the first and second terms, respectively, on the right-hand side of the modified Landau-Lifshitz equation of motion for magnetization for one-dimensional current flow along the $x$ direction,

$$
\frac{\partial \mathbf{m}}{\partial t}+\gamma_{0} \mathbf{m} \times \mathbf{H}_{\mathrm{eff}}-\alpha \mathbf{m} \times \frac{\partial \mathbf{m}}{\partial t}=-u \frac{\partial \mathbf{m}}{\partial x}+\beta u\left(\mathbf{m} \times \frac{\partial \mathbf{m}}{\partial x}\right)
$$

where $u=J P g \mu_{\mathrm{B}} / 2 e M_{\mathrm{s}}$ is an effective drift velocity for the spin current, $J$ is the current density, $P$ is the spin polarization, $g$ is the gyromagnetic ratio, $\mu_{\mathrm{B}}$ is the Bohr magneton, $e$ is the electron charge and $M_{\mathrm{s}}$ is the saturation magnetization. In addition, $\mathbf{m}$ is a unit vector representing the orientation of magnetization, $\gamma_{0}$ is the gyromagnetic factor, $\mathbf{H}_{\text {eff }}$ is an effective field, $\alpha$ is the Gilbert damping constant and $\beta$ is the non-adiabatic coefficient.

The CoNi film structure consists of a $\mathrm{Si} / \mathrm{SiO}_{2} / \mathrm{Ta} / \mathrm{Pd} /$ reference layer $/ \mathrm{Cu} /$ free layer/Ta multilayer film, grown by sputtering. The reference layer is a composite $\left[(\mathrm{Co} / \mathrm{Pd})_{2} /(\mathrm{Co} / \mathrm{Ni})_{3}\right]$ to enhance the perpendicular magnetic anisotropy, whereas the free layer is a $\left[(\mathrm{Co} / \mathrm{Ni})_{4} /(\mathrm{Co} / \mathrm{Pd})\right]$ multi-layer with a lower anisotropy. The resulting films are highly (111) textured. The use of Pd allows us to get higher uniformity and lower distribution of intrinsic pinning fields than our previous spin valves based on $[\mathrm{Co} / \mathrm{Pt}] /[\mathrm{Co} / \mathrm{Ni}]$ multilayers ${ }^{18}$. In addition, higher giant magnetoresistance ratios are obtained, with values varying from 3.7 to $4.5 \%$. The FePt-based spin valves consist of a $\mathrm{MgO}(001) /$ free layer/Pt/reference layer multilayer film grown by molecular beam epitaxy at high temperature. Both the reference and the free layers consist of a high chemically ordered $\mathrm{L}_{10} \mathrm{FePt}$, with a very high anisotropy $\left(B_{\mathrm{a}} \sim 10 \mathrm{~T}\right)$ along the $(001)$ direction. As the microstructures are different in the FePt underlayer and overlayer, the electrodes have slightly different switching fields ${ }^{23}$. The typical magnetoresistance ratio in the FePt-based spin valves is about $0.8 \%$ at room temperature. The CoNi free layer has narrow domain walls $\left(\lambda=\sqrt{A / K_{\text {eff }}} \sim 10 \mathrm{~nm}\right.$, where $K_{\text {eff }}=K_{\mathrm{u}}-\mu_{0} M_{\mathrm{s}}^{2} / 2$ with $K_{\mathrm{u}}$ being the uniaxial anisotropy) and low coercivity $(<0.5 \mathrm{kOe})$, whereas the FePt free layer has an even stronger perpendicular magnetic anisotropy, giving rise to ultra-narrow domain walls $(\lambda \sim 1.2 \mathrm{~nm})$, but with larger coercive fields ( $>4 \mathrm{kOe}$ )

High-quality 200-nm-wide wires with low edge roughness $(<10 \mathrm{~nm})$ were fabricated by means of conventional electron beam lithography and ion milling. A large magnetic area was added at one end of the magnetic wires that acts as a domain wall reservoir. For the CoNi devices, we have lithographically defined artificial constrictions with a central part of $100 \mathrm{~nm}$ and lateral size varying from 10 to $500 \mathrm{~nm}$. To measure the giant magnetoresistance, large electrical electrodes were patterned using optical lithography. For the CoNi and FePt devices, up to 10 wires with different constriction shapes and structural defects were measured.

For the transport measurements, the giant magnetoresistance signal at room temperature is obtained using an a.c. $(10 \mu \mathrm{A})$ lock-in technique. For measurements on CoNi samples, the d.c. current was injected using a bias-tee. For measurements on FePt samples, the current source used provided directly the sum of a d.c. and an a.c. current. The giant magnetoresistance signal was measured as a function of time to determine the depinning time under magnetic field and/or current. The measurement procedure is conducted as follows. First, the hard and free layers of the spin valve are saturated with a strong negative field. Then a constant positive field is applied to nucleate in the free layer and inject a single domain wall into the wire while measuring the giant magnetoresistance as a function of time. A d.c. current is applied in addition to the positive field to facilitate wall depinning. The typical time resolutions of our set-up are $50 \mathrm{~ms}(\mathrm{CoNi})$ and $100 \mathrm{~ms}(\mathrm{FePt})$, and the spatial resolution is about $100 \mathrm{~nm}$. However, the rise time of the electromagnet (typically $0.5-1 \mathrm{~s}$ ) gives an upper bound to the magnetic fields we can apply, which also places a lower bound on the depinning times we can measure. Joule heating under d.c. currents in the CoNi samples has been measured and is found to be up to $20 \mathrm{~K}$ at the maximum used current density of $2 \times 10^{7} \mathrm{~A} \mathrm{~cm}^{-2}$. For the FePt system, the Joule heating has been measured using measurement of the wire resistivity, and is below $5 \mathrm{~K}$ for all current densities presented in this letter. In all samples, the current density has been calculated by supposing a homogeneous current flowing through the whole stack, so the given $J$ value is an upper estimate of the current density flowing in the free layer. The range of applied currents has been limited in the present study to limit deterioration of our samples due to electrical discharge and to minimize the effects of Joule heating. The latter is a significant issue for data analysis, as even changes of a few tens of kelvins can mask the effects of spin-transfer.

For the fits to the cumulative distribution function, we typically find a $1 \%$ error in the characteristic time $\tau$ for activation over a single barrier process in $\mathrm{CoNi}$, with a similar figure for activation processes in FePt after we have accounted for the most probable path, as discussed elsewhere ${ }^{24}$.

For the micromagnetics studies, we studied a single FePt thin film in which the domain wall is pinned on a single defect to minimize the simulation time. This simple system, which models the free layer of the FePt-based spin valves, captures the essential physics of stochastic domain-wall depinning. For this calculation, the thermal fluctuations and the adiabatic and non-adiabatic contributions of the spin transfer have been included in the modified Landau-Lifshitz-Gilbert equation. The simulated system consists of a FePt film $80 \times 50 \times 5 \mathrm{~nm}^{3}$ in size with a uniaxial anisotropy $K_{u}$, except at the centre where a defect of size $5 \times 10 \times 5 \mathrm{~nm}^{3}$ is introduced with a lower anisotropy constant $K_{\mathrm{def}}=K_{\mathrm{u}} / 2$.

For the analytic theory, we derived the field- and current-dependent Arrhenius transition rate from the stochastic equations of motion for a one-dimensional Bloch wall ${ }^{26}$. By solving for the stationary distribution function using a Fokker-Planck formalism, we followed Kramers's approach to obtain an analytical expression for the characteristic attempt frequency and changes to the energy barrier due to spin-torques. We find that the barrier energy changes linearly with current, $E_{\mathrm{b}}=E_{\mathrm{b}, 0}-\sigma I$, where $\sigma$ represents an efficiency factor that is proportional to the non-adiabatic coefficient and $I$ is the applied current,

$$
\sigma=\beta P \frac{h}{e} \frac{\Delta x}{\lambda}
$$

where $\Delta x$ is the width of the pinning potential (as seen by the domain wall) and $\lambda$ is the domain-wall width. The experimental values of beta are determined by fitting the slopes of $\ln \tau$ as a function of current and by assuming that $\Delta x \approx \lambda$. It can be shown that the width of the potential well due to a pinning site has a weak dependence on the applied field, which results in a weak field dependence of the efficiency parameter $\sigma$.

Received 21 May 2009; accepted 23 September 2009; published online 8 November 2009

\section{References}

1. Berger, L. Exchange interaction between ferromagnetic domain wall and electric current in very thin metallic films. J. Appl. Phys. 55, 1954-1956 (1984).

2. Tatara, G. \& Kohno, H. Theory of current-driven domain wall motion: Spin transfer versus momentum transfer. Phys. Rev. Lett. 92, 086601 (2004).

3. Zhang, S. \& Li, Z. Roles of nonequilibrium conduction electrons on the magnetization dynamics of ferromagnets. Phys. Rev. Lett. 93, 127204 (2004).

4. Waintal, X. \& Viret, M. Current-induced distortion of a magnetic domain wall. Europhys. Lett. 65, 427-433 (2004).

5. Xiao, J., Zangwill, A. \& Stiles, M. D. Spin-transfer torque for continuously variable magnetization. Phys. Rev. B 73, 054428 (2006)

6. Vanhaverbeke, A. \& Viret, M. Simple model of current-induced spin torque in domain walls. Phys. Rev. B 75, 024411 (2007).

7. Tatara, G., Kohno, H. \& Shibata, J. Theory of domain wall dynamics under current. J. Phys. Soc. Jpn 77, 031003 (2008).

8. Freitas, P. P. \& Berger, L. Observation of $\mathrm{s}-\mathrm{d}$ exchange force between domain walls and electric current in very thin permalloy films. J. Appl. Phys. 57, 1266-1269 (1985).

9. Grollier, J. et al. Switching a spin valve back and forth by current-induced domain wall motion. Appl. Phys. Lett. 83, 509-511 (2003).

10. Kimura, T., Otani, Y., Yagi, I., Tsukagoshi, K. \& Aoyagi, Y. Suppressed pinning field of a trapped domain wall due to direct current injection. J. Appl. Phys. 94, 7266-7269 (2003).

11. Klaui, M. et al. Domain wall motion induced by spin polarized currents in ferromagnetic ring structures. Appl. Phys. Lett. 83, 105-107 (2003).

12. Yamanouchi, M., Chiba, D., Matsukura, F. \& Ohno, H. Current-induced domain-wall switching in a ferromagnetic semiconductor structure. Nature 428, 539-542 (2004).

13. Ravelosona, D., Lacour, D., Katine, J. A., Terris, B. D. \& Chappert, C. Nanometer scale observation of high efficiency thermally assisted current-driven domain wall depinning. Phys. Rev. Lett. 95, 117203 (2005). 
14. Beach, G. S. D., Knutson, C., Nistor, C., Tsoi, M. \& Erskine, J. L. Nonlinear domain-wall velocity enhancement by spin-polarized electric current. Phys. Rev. Lett. 97, 057203 (2006).

15. Hayashi, M. et al. Influence of current on field-driven domain wall motion in permalloy nanowires from time resolved measurements of anisotropic magnetoresistance. Phys. Rev. Lett. 96, 197207 (2006).

16. Laribi, S. et al. Reversible and irreversible current induced domain wall motion in CoFeB based spin valves stripes. Appl. Phys. Lett. 90, 232505 (2007).

17. Meier, G. et al. Direct imaging of stochastic domain-wall motion driven by nanosecond current pulses. Phys. Rev. Lett. 98, 187202 (2007).

18. Burrowes, C. et al. Role of pinning in current driven domain wall motion in wires with perpendicular anisotropy. Appl. Phys. Lett. 93, 172513 (2008).

19. Boulle, O. et al. Nonadiabatic spin transfer torque in high anisotropy magnetic nanowires with narrow domain walls. Phys. Rev. Lett. 101, 216601 (2008).

20. Allwood, D. A. et al. Magnetic domain-wall logic. Science 309, 1688-1692 (2005).

21. Parkin, S. S. P., Hayashi, M. \& Thomas, L. Magnetic domain-wall racetrack memory. Science 320, 190-194 (2008)

22. Thiaville, A., Nakatani, Y., Miltat, J. \& Suzuki, Y. Micromagnetic understanding of current-driven domain wall motion in patterned nanowires. Europhys. Lett. 69, 990-996 (2005).

23. Mihai, A. P. et al. Magnetization reversal dominated by domain wall pinning in FePt based spin valves. Appl. Phys. Lett. 94, 122509 (2009).

24. Attané, J. P., Ravelosona, D., Marty, A., Samson, Y. \& Chappert, C. Thermally activated depinning of a narrow domain wall from a single defect. Phys. Rev. Lett. 96, 147204 (2006).

25. Martinez, E., Lopez-Diaz, L., Alejos, O., Torres, L. \& Tristan, C. Thermal effects on domain wall depinning from a single notch. Phys. Rev. Lett. 98, 267202 (2007).

26. Kim, J.-V. \& Burrowes, C. Influence of magnetic viscosity on domain wall dynamics under spin-polarized currents. Preprint at $<$ http://arxiv.org/abs/0907.2867vl> (2009).
27. Metaxas, P. J. et al. Creep and flow regimes of magnetic domain-wall motion in ultrathin $\mathrm{Pt} / \mathrm{Co} / \mathrm{Pt}$ films with perpendicular anisotropy. Phys. Rev. Lett. 99, 217208 (2007).

28. Le Maho, Y., Kim, J.-V. \& Tatara, G. Spin-wave contributions to current-induced domain wall dynamics. Phys. Rev. B 79, 174404 (2009).

29. Barnes, S. E. \& Maekawa, S. Current-spin coupling for ferromagnetic domain walls in fine wires. Phys. Rev. Lett. 95, 107204 (2005).

30. Hayashi, M., Thomas, L., Moriya, R., Rettner, C. \& Parkin, S. S. P. Current-controlled magnetic domain-wall nanowire shift register. Science 320, 209-211 (2008).

\section{Acknowledgements}

We acknowledge fruitful discussions with Y. Le Maho and invaluable aid from D. Stanescu, V. Mathet (CTU IEF-MINERVE) and J.-C. Pillet for the device fabrication. This work was supported by the ISTRADE contract from the French National Research Agency (ANR). C.B. acknowledges financial support from C'Nano IDF.

\section{Author contributions}

C.B. and A.P.M. contributed in equal parts to this work. I.T. and E.E.F. grew the $\mathrm{CoNi}$ films. C.B. did the lithography, carried out the experiments, analysed and interpreted the data for the CoNi samples. A.P.M., L.V., A.M. and J.-P.A. carried out the experiments, analysed and interpreted the data for the FePt samples. J.-V.K. developed the theory and prepared the manuscript. F.G.-S. and L.D.B.-P. did the micromagnetics simulations. L.V., A.M., J.-P.A., D.R., E.E.F. and L.D.B.-P. edited and commented on the manuscript. Y.S., C.C., D.R. and J.-P.A. planned the project. D.R., L.B. and J.-P.A. managed the project. D.R. coordinated the work within the ISTRADE contract.

\section{Additional information}

The authors declare no competing financial interests. Supplementary information accompanies this paper on www.nature.com/naturephysics. Reprints and permissions information is available online at http://npg.nature.com/reprintsandpermissions. Correspondence and requests for materials should be addressed to D.R. 